\title{
Controlling a Single DNA Molecule in an Electric Field by Means of In Situ Atomic Force Microscopy
}

\author{
Jae Sun Jeong and Giovanni Dietler ${ }^{\mathrm{Z}}$ \\ Laboratory of Physics of Living Matter, EPFL, Lausanne 1015, Switzerland
}

DNA is the bio-polymer containing the genetic information needed for the development and functioning for all living organisms. It has a polymeric structure consisting of units called nucleotides, each consisting of a non-polar, hydrophobic interior (the base pairs) and polar, hydrophilic exterior which is negatively charged due to the phosphate groups along the backbone of the DNA. Its heterogeneous properties permit DNA molecule to interact with other molecules and different types of substrates at the same time. It is important to understand the DNA morphology on flat surface that serves as a template for DNA based sensors and microarray applications, particularly under an electric field. Taken together, the ability to deposit a single DNA molecule on the electrode is essential to increase its specificity. In our study, we optimize the conditions in order to control a single DNA molecule adsorbing and desorbing to/from the electrode. Simultaneously dynamic imaging enables us to analyze its morphological behavior by real-time Electrochemical Atomic Force Microscopy (EC-AFM) in aqueous conditions.

(C) 2012 The Electrochemical Society. [DOI: 10.1149/2.008207jes] All rights reserved.

Manuscript submitted January 31, 2012; revised manuscript received March 14, 2012. Published July 17, 2012. This was Paper 1414 presented at the Seattle, Washington, Meeting of the Society, May 6-10, 2012.

Atomic force microscopy (AFM) is a powerful single molecule imaging technique for biomolecules which enables exploration of the detailed topography at molecular level. Moreover, coupled with the ability to characterize living bio-materials under physiological conditions, the AFM data provide information for statistical analysis of the biomolecule's conformation from AFM images. In recent studies, an electrochemical cell combined with an AFM (EC-AFM) has been used to visualize the surface conformation of DNA with nano-meter size resolution under electric field in-situ (AFM imaging in solution during electrochemical reaction). ${ }^{1,2}$ This technical development allows us to visualize the dynamic movement of DNA molecule in an electric field without perturbing its bio-functionality. There are many reports regarding DNA network film adsorption on a highly oriented pyrolytic graphite (HOPG) electrode under controlled potential with an ex-situ AFM..$^{3-5}$ However, until now there is no report of in-situ single DNA molecule immobilization or release by applying potential through EC-AFM.

In our study, we optimize the conditions for immobilization/release of a single DNA molecule to/from a HOPG electrode in an electric field. The electrode, by the applied potential, enables the negatively charged phosphate backbone group of DNA molecule to be attracted toward electrode, or repel DNA molecule from negatively charged electrode by electrostatic interaction. Then the morphological changes of DNA molecules on the electrode depending on the applied potential were investigated using in-situ EC-AFM. Commonly, it is not easy to obtain a stable conformation of DNA molecules in aqueous condition because of the thermal agitation that allows DNA molecules to move in electrolyte solution. However the electrostatic interaction force between molecules and electrode was reinforced during the DNA immobilization process by an applied potential, yielding the increased stability. ${ }^{3-5}$ It is critical to understand the morphological and electrochemical properties of DNA molecules in order to construct the DNA based electrochemical biosensor. ${ }^{6}$ We report here for the first time a single DNA molecule immobilization and release in aqueous condition on HOPG electrode in-situ EC-AFM by tuning the electric potential. This opens new possibilities for fundamental investigation toward future DNA-based biological sensors. ${ }^{7}$

\section{Experimental}

Materials. - Double stranded linear DNA (Lambda Mix Marker 19) was purchased from Fermentas and used without any purification process. It was dissolved in phosphate buffered saline (Sigma-Aldrich) resulting in $\mathrm{pH}$ 7.4, $0.01 \mathrm{M}$ phosphate buffer (PBS). The final concentration of DNA sample was adjusted to 1,4 and $5 \mu \mathrm{g} / \mathrm{mL}$ in $\mathrm{pH} 7.4$,

${ }^{\mathrm{z} E-m a i l: ~ g i o v a n n i . d i e t l e r @ e p f l . c h ~}$
0.01 M PBS. Highly oriented pyrolytic graphite (HOPG, ZYA grade, $10 \times 10 \times 1.5 \mathrm{~mm}$ ) was purchased from NT-MDT (Russia) and used as a substrate, and as the working electrode (WE). HOPG was cleaved freshly with an adhesive tape before use. We used a platinum $(\mathrm{Pt})$ wire (0.25 mm diameter, Sigma-Aldrich) as a counter electrode (CE) and a silver $(\mathrm{Ag})$ wire $(0.50 \mathrm{~mm}$ diameter, Sigma-Aldrich $)$ as the reference electrode (RE), also known as pseudo - reference electrode. Prior to use, the Ag wire was polished with a sand paper and the Pt wire was sonicated in purified water followed by rinsing with purified water.

Electrochemical system combined with an AFM (EC-AFM).Scheme 1 illustrates the one compartment cell for EC-AFM consisting of three electrodes: Pt (CE), Ag (RE) and HOPG (WE) mounted in AFM fluid cell (MMTMEC, Veeco). The potential control was made with the potentiostat (AMETEK 263A, Princeton Applied Research, USA) using the POWER PULSE software. Differential pulse voltammetry measurements were conducted with another potentiostat (Model 2053, Amel Instrument, Italy) and the obtained voltammograms were smoothed by means of the Adjacent-Averaging algorithm. HOPG substrate was compressed against the cell with a silicon O-ring to obtain a good seal in order to prevent liquid leakage during experiments. AFM (Veeco, Santa Barbara, USA) was used to visualize a single molecule with silicon nitride coated V-shape AFM probes (Veeco): the probes had a resonance frequency range of $9-10 \mathrm{kHz}$ and a spring constant of $0.08 \mathrm{~N} / \mathrm{m}$ for liquid tapping mode. All electrochemical studies and AFM imaging were carried out in PBS in order to keep the physiological conditions constant. The statistical properties of DNA were measured from AFM images with Nanoscope IIIa software. The obtained AFM images were simply flattened with the built-in software; the background noise and brightness were adjusted.

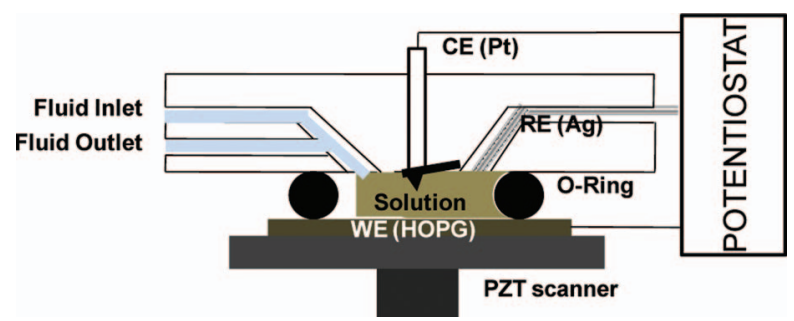

Scheme 1. Illustration of the one compartment cell for electrochemical AFM (EC-AFM) consisting of three electrodes: working electrode (WE, HOPG), reference electrode (RE, Ag wire) and counter electrode (CE, Pt wire) mounted in an AFM fluid cell. 

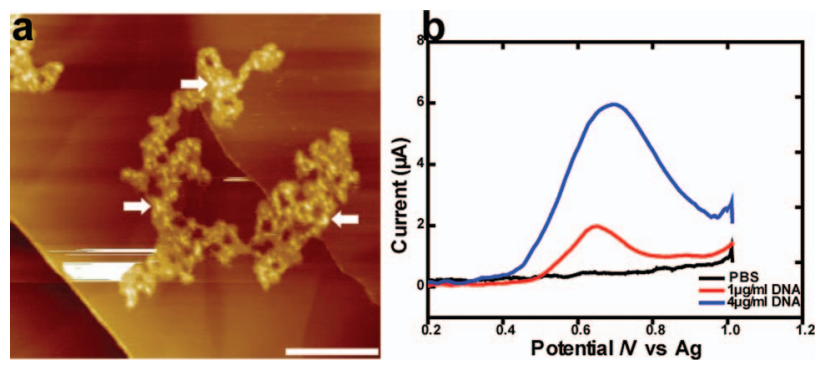

Figure 1. (a) AFM topographical images of $\lambda$-DNA molecules in aqueous condition after $5 \mathrm{~min}$ free adsorption from $30 \mu \mathrm{L}$ of $5 \mu \mathrm{g} / \mathrm{mL}$ in $\mathrm{pH} 7.1$, $0.1 \mathrm{M}$ PBS on HOPG (z scale: $10 \mathrm{~nm}$, scale bar: $250 \mathrm{~nm}$ ), (b) Differential pulse voltammetry graph obtained in the EC-AFM cell with three-electrode: $1 \mu \mathrm{g} / \mathrm{mL}$ (red), $4 \mu \mathrm{g} / \mathrm{mL}$ (blue) of $\lambda$-DNA in pH 7.1, 0.1 M PBS electrolyte (black).

\section{Results and Discussion}

Globular aggregates of DNA were formed on bare HOPG.- As a control experiment, $30 \mu \mathrm{L}$ of $5 \mu \mathrm{g} / \mathrm{mL} \lambda$-DNA in PBS were injected through fluid inlet in the AFM fluid cell. The DNA solution was kept inside the cell for $5 \mathrm{~min}$ on bare HOPG for free adsorption. The electrochemical cell was flushed prior imaging and no air bubbles were present. After $5 \mathrm{~min}$, AFM imaging (Fig. 1a) showed the immobilized $\lambda$-DNA on HOPG in aqueous condition. Globular aggregates of DNA (arrows, height: $1.87 \pm 0.35 \mathrm{~nm}$ ) were observed on bare HOPG. It is possible that the salt content in PBS, mainly $\mathrm{NaCl}$, could partially interrupt the adsorption process by shielding the anionic phosphate backbone group of DNA. Moreover, the observed DNA aggregates from free adsorption are of limited use for further biological sensor application because of inaccessibility of the DNA sequence. To immobilize a single DNA molecule, we applied a positive potential to the HOPG electrode. In order to avoid the DNA oxidation by applying a potential, we first determined the oxidation peak of $\lambda$-DNA on HOPG (Fig. 1b) and it was found to be between $0.4 \mathrm{~V}$ and $1.0 \mathrm{~V}$ at two different concentrations. Thus, for deposition of DNA we applied a positive potential in the range from $0 \mathrm{mV}$ to $300 \mathrm{mV}$.

Electrostatic force induces the deposition of linear DNA strands when a positive potential is applied.- Samples were prepared under the same procedure as described above with an applied positive potential in the range from $0 \mathrm{mV}$ to $300 \mathrm{mV}$ to the HOPG electrode for 5 minutes. Simultaneously, DNA conformation was investigated insitu at different applied potential as shown in Fig. 2. At $0 \mathrm{mV}$, most of the globular aggregates of $\lambda$-DNA molecules as seen in Fig. 1a disappeared and linear strands with island shape as marked by asterisk were observed (Fig. 2a). Although they were immobilized without any potential, a minimum current flow $(-9.6 \mathrm{nA})$ was present in PBS and enabled to form linear strands of DNA. It is likely that the hydrophobic and electrostatic interactions co-exist in the PBS electrolyte and that aggregates and linear DNA form at same time. At $50 \mathrm{mV}$, a linear strand (arrow, height: $1.57 \pm 0.22 \mathrm{~nm}$ ) divided into two strands with half height $(0.74 \pm 0.16 \mathrm{~nm})$ and exhibiting an alignment along the three-fold symmetry of HOPG (circle) in Fig. 2b. It is possible that multiple sites on the negatively charged phosphate backbone group of DNA can bind to the graphite lamellar structure through electrostatic attraction at $50 \mathrm{mV}$ and subsequently the hydrogen bonds between DNA base pairs break resulting in divided thin linear strands. In accordance, we also observed that several thin linear strands of DNA were separated at the end of the thick linear DNA as marked by asterisk in Fig. 2b. The adsorbed DNA molecules followed the three-fold symmetry of HOPG structure and permit us to envision that electrostatic force starts to govern the interaction between molecules and HOPG electrode when a potential is applied rather than hydrophobic interaction. Fig. $2 \mathrm{c}$ shows the adsorbed linear $\lambda$-DNA molecules (height: $1.81 \pm 0.42 \mathrm{~nm}$ ) at $100 \mathrm{mV}$ potential. When $300 \mathrm{mV}$ were applied, most small aggregation islands disappeared (Fig. 2d) and a

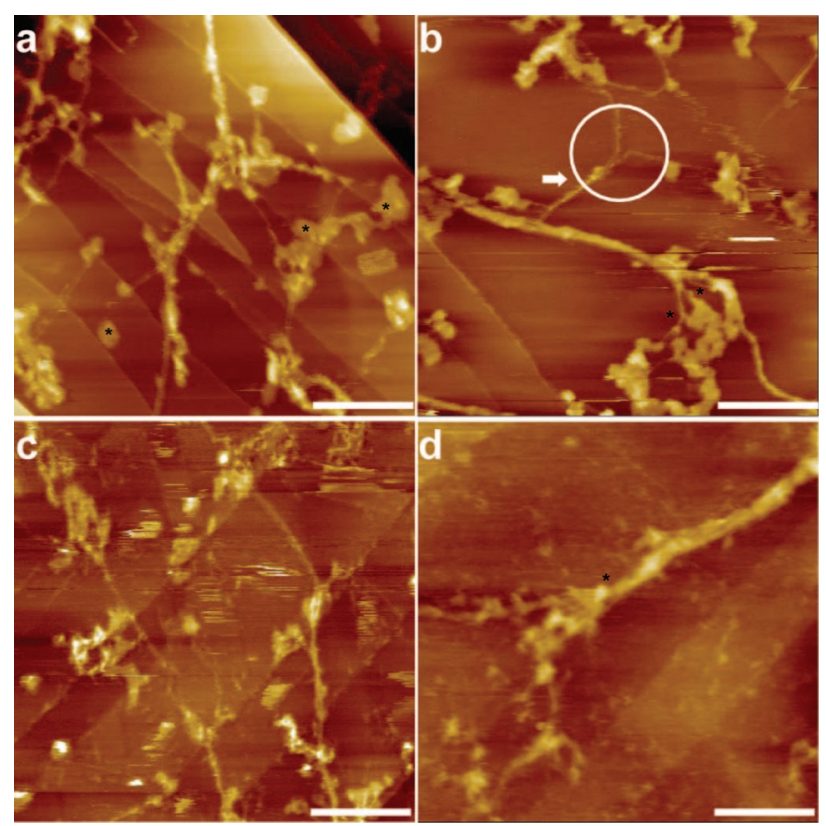

Figure 2. (a) AFM topographical images of $\lambda$-DNA molecules in aqueous condition on HOPG by applying $0 \mathrm{mV}$, (b) $50 \mathrm{mV}$, (c) $100 \mathrm{mV}$, (d) $300 \mathrm{mV}$ (vs Ag wire). (z scale: $7 \mathrm{~nm}$, scale bar: $250 \mathrm{~nm}$ )

thick DNA strand (height: $2.02 \pm 0.37 \mathrm{~nm}$ ) was observed revealing that several single strands joined together as marked by asterisk. We note that a positively charged electrode attracts DNA molecules toward HOPG electrode causing wide linear strands with height $(\sim 2 \mathrm{~nm})$ at any potential. Thus, we sought the optimal conditions to adsorb single DNA molecules in aqueous solutions for the purpose of applying this method for biological sensors. Previously, it was reported that the DNA morphology was affected by the DNA solution concentration and by the adsorption time. ${ }^{3}$ By controlling these two factors, we were able to obtain a single $\lambda$-DNA molecule (height: $1.78 \pm 0.3 \mathrm{~nm}$ ) on the HOPG electrode when the DNA concentration was lowered $(1 \mu \mathrm{g} / \mathrm{mL})$ and the adsorption time shortened $(1 \mathrm{~min})$ at the same potential of $300 \mathrm{mV}$. Fig. 3a shows that the linear $\lambda$-DNA molecule (arrows) has approximately $5 \mu \mathrm{m}$ length: our solution contains a wide range of $\lambda$-DNA fragment lengths up to the maximum $15 \mu \mathrm{m}$ length of the whole $\lambda$-DNA. The DNA molecules were immobilized along to edge-plane of HOPG since it is well known that the edge-plane sites of HOPG are more active than basal plane ${ }^{8}$ for electrochemical reaction. Fig. 3b shows a high resolution image of the area indicated in Fig. 3a exhibiting a single linear DNA strand of approximately $1.78 \mathrm{~nm}$ height which is in good agreement with the

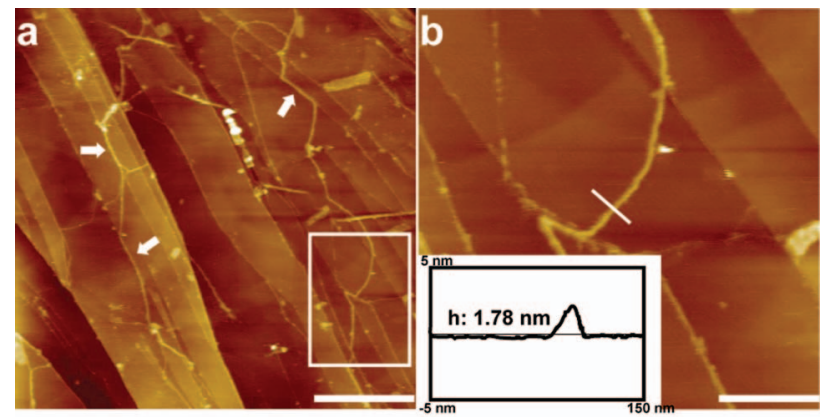

Figure 3. (a) AFM topographical image of $\lambda$-DNA molecules in PBS on HOPG after applying $300 \mathrm{mV}$ for $1 \mathrm{~min}$ from $1 \mu \mathrm{g} / \mathrm{mL}$ in $\mathrm{pH} 7.10 .1 \mathrm{M}$ PBS solution, (b) Zoom of the area indicated in (a) (inset: cross-section profile through the white line in image (b)). (z scale: $10 \mathrm{~nm}$, scale bar: (a) $1 \mu \mathrm{m}$, (b) $250 \mathrm{~nm}$ ) 

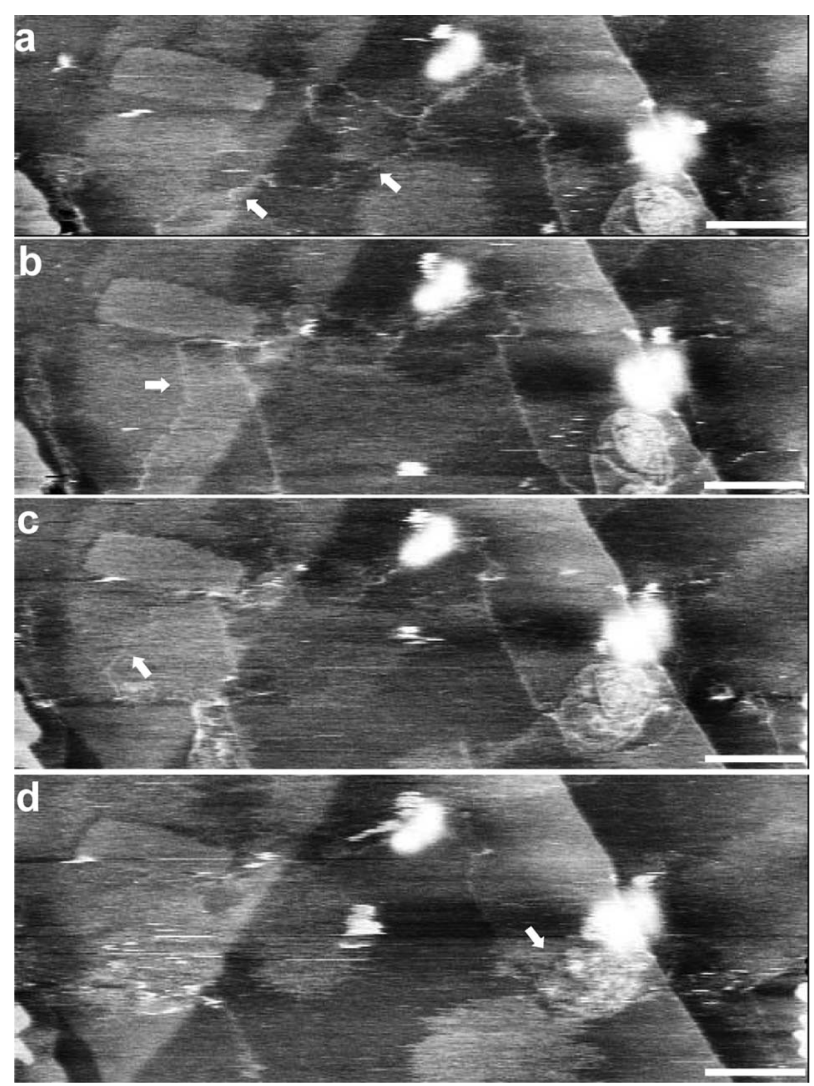

Figure 4. Time-series of AFM topographical images of $\lambda$-DNA molecules in aqueous condition on dodecylamine modified HOPG at an applied potential of (a) $0 \mathrm{mV}$, (b) $-10 \mathrm{mV}$, (c) $-20 \mathrm{mV}$, (d) $-30 \mathrm{mV}$ for $5 \mathrm{~min}$. (z scale: $5 \mathrm{~nm}$, scale bar: $125 \mathrm{~nm}$ ).

crystallographic value for single DNA molecules. ${ }^{9-12}$ The height of $\lambda$-DNA in liquid is $1.5-2 \mathrm{~nm}$ on mica ${ }^{9-12}$ and it is always smaller than the crystallographic value of DNA $(2.0 \mathrm{~nm})$ because of the elastic deformation induced by the tip-sample interaction. ${ }^{10}$ The measured height of linear $\lambda$-DNA here was also reduced by tip-sample interaction in liquid. We conclude that shortening the adsorption time leads to single DNA molecule and avoids the interaction between adjacent DNA molecules that otherwise results in wide linear strands of DNA (compare Figs. 2d to 3b).

The DNA molecules can be desorbed from the electrode by an applied negative potential. - After the deposition of a single $\lambda$-DNA molecule by a positive potential, we turned to the negative potential to release the adsorbed $\lambda$-DNA molecules from the solid surface. However, we found that it required much higher negative potential $(>-300 \mathrm{mV})$ because they were already strongly attached to the electrode by positive potential $(+300 \mathrm{mV})$. The application of high potential caused the electronic interference with the AFM imaging process which made impossible to obtain images. Therefore, we employed dodecylamine modified HOPG (DA-HOPG) to give a certain distance between $\lambda$-DNA and the electrode for easy release with low potential. We have also tested graphite modifier (GM) treated HOPG as substrate, yet it turned out that GM-HOPG was not stable in aque- ous conditions in our electrochemical cell. For better observation of $\lambda$-DNA molecules and its stable arrangement on the HOPG substrate, here we used an amphilphilic dodecylamine (DA) layer which has two functional groups at its both ends; an alkyl chain for graphite and an amine group for DNA affinity. ${ }^{13} \mathrm{~A}$ set of data illustrating the release of $\lambda$-DNA molecule from the DA-HOPG was recorded in Fig. 4. First $30 \mu \mathrm{L}$ of $5 \mu \mathrm{g} / \mathrm{mL} \lambda$-DNA was deposited on DA-HOPG for $30 \mathrm{sec}$ and the excess $\lambda$-DNA was rinsed with pure water followed by drying with air. Then the sample was bathed in PBS and imaged in tapping mode as seen in Fig. 4a. When $-10 \mathrm{mV}$ potential was applied, the DNA molecule (arrow in Fig. 4a) was displaced upward as seen in Fig. 4b (arrow). When $-20 \mathrm{mV}$ potential was applied, the other part of the DNA molecule was moved as indicated with the arrow in Fig. 4c. Most of DNA molecules were finally desorbed at $-30 \mathrm{mV}$ potential except the coiled DNA molecule (arrow in Fig. 4d) which remained on the surface due to the strong interaction with HOPG. With repeated this series of experiments, and found that the potential between $-20 \mathrm{mV}$ and $-30 \mathrm{mV}$ was enough to repel a single DNA molecule from the negatively charged surface. The stability of the dodecylamine layer was confirmed in our control experiments at applied potentials as above $(-30 \mathrm{mV})$ and within the same time intervals.

\section{Conclusions}

Single DNA molecule studies are essential to investigate their behavior in an electric field and especially in aqueous conditions in order to preserve their biological function properly. In particular, in-situ AFM imaging without dehydration of DNA molecules is a prerequisite to accurately investigate the behavior in an electric field. Our results demonstrate that a single DNA molecule can be immobilized to positively charged HOPG by an applied positive potential of 300 $\mathrm{mV}$ (vs. Ag wire) by controlling the DNA concentration in solution $(1 \mu \mathrm{g} / \mathrm{mL}$ in PBS) and the adsorption time $(1 \mathrm{~min})$. Moreover, the negative phosphate backbone groups of $\lambda$-DNA molecules were repelled by the negatively charged HOPG surface: a $-25 \mathrm{mV}$ negative potential was enough to desorb the DNA molecules. Our results lead to a better understanding of DNA behavior in an electric field which provides new insights and could lead to further advances in biological sensor applications.

\section{Acknowledgment}

This research was supported by National Competence Center in Biomedical Imaging (NCCBI).

\section{References}

1. G. M. Brown, T. Thundat, D. P. Allison, and R. J. Warmack, J. Vac. Sci. Technol. A, 10, 3001 (1992)

2. J. P. Bearinger, C. A. Orme, and J. L. Gilbert, Surf. Sci., 491, 370 (2001).

3. X. Jiang and X. Lin, Electrochemistry Communications, 6, 873 (2004).

4. A. M. O. Brett and A-M C. Paquim, Bioelectrochem., 66, 117 (2005).

5. A. M. O. Brett and A-M Chiorces, Langmuir, 19, 3830 (2003).

6. V. C. Diculescu, A. M. C. Paquim, and A. M. O. Brett, Sensors, 5, 377 (2005).

7. T. G. Drummond, M. G. Hill, and J. K. Barton, Nature. Biotech., 21, 1192 (2003).

8. M. I. Pividori and S. Alegret, Top. Curr. Chem., 260, 1 (2006).

9. F. Kienberger, L. T. Costa, R. Zhu, G. Kada, M. Reithmayer, L. Chtcheglova, C. Rankl, A. B. F. Pacheco, S. Thalhammer, V. Pastushenko, W. M. Heckl, D. Blaas, and P. Hinterdorfer, Biomaterials, 28, 2403 (2007).

10. F. M-Herrero, J. Colchero, and A. M. Baró, Ultramicroscopy, 96, 167 (2003).

11. J. Mou, D. M. Czajkowsky, Y. Zhang, and Z. Shao, FEBS Letters, 371, 279 (1995).

12. Y. L. Lyubchenko and L. S. Shlyakhtenko, Proc. Natl. Acad. Sci., 94, 496 (1997).

13. J. Adamcik, S. Tobenas, G. D. Santo, D. Klinov, and G. Dietler, Langmuir, 25, 3159 (2009). 